\title{
A review of economic risking methods commonly used in hydrocarbon exploration
}

\author{
I. Lerche ${ }^{1}(\mathbb{C}$
}

Received: 10 June 2018 / Accepted: 13 December 2018 / Published online: 20 December 2018

(c) The Author(s) 2018

\begin{abstract}
Three different risking methods for hydrocarbon exploration projects are discussed. The so-called Cozzolino procedure is shown to have the major drawback that it predicts less and less involvement should be taken in a project the greater the potential gains. The Kelly procedure circumvents this weakness but has itself the weakness that it can only allow involvement in a project when the expected value is positive despite the fact that the expected value has a standard deviation that allows for a positive domain around a negative expected value. The corporate confidence risk assessment allows for involvement in a project even when the expected value is negative, but has the weakness that there are some ad hoc factors involved that, nevertheless, permit a practical approach to obtain allowing a corporation to assess the confidence the corporation has in an exploration assessment. Numerical illustrations are provided to show how the Kelly procedure and the corporate confidence procedure operate for both single and multiple projects. Only some simple examples are given for the Cozzolino method for which an exhaustive investigation has been given elsewhere in other publications, demonstrating the major flaws. The search for a more robust risking method is ongoing.
\end{abstract}

Keywords Hydrocarbon exploration $\cdot$ Economic risk $\cdot$ Corporate confidence $\cdot$ Cozzolino $\cdot$ Kelly

\section{Preamble}

The search for economic oil and gas reserves is an extremely expensive undertaking. Depending on the location (in particular, onshore versus offshore drilling), exploration wells can cost from the low millions of dollars to hundreds of million dollars with no guarantee of finding economically producible reserves. These high costs mandate that one estimate the probable potential gains ahead of drilling so that one can assess the likely corporate gain or loss attendant upon drilling. There is then an economic risk attached to each potential hydrocarbon reservoir prior to drilling.

This review is concerned with three economic risking methods commonly used in such evaluations. The points to make are several. First, prior to using any economic risk procedure, one should be aware of the basic assumptions underlying each method. Failure to do so can lead one to situations

I. Lerche

lercheian@yahoo.com

1 Institut für Geowissenschaften, Naturwissenschaftliche Fakultät III, Martin-Luther Universität, 06099 Halle, Germany where one blindly trusts the results of an evaluation without giving any thought to the correctness and appropriateness of the results. Second, most economic risk applications are performed numerically. The worth of any results is then firmly tied to the correctness and accuracy of the numerical program used. While a numerical program always gives precise results, the question of whether the results obtained also accurately reflect the economic assessment depends on the manner in which the program was written. One cannot overstate too strongly that one should provide analytically solvable illustrations and compare their results against the results of numerical programs obtained under the same conditions. Often, such comparisons uncover errors of omission or commission in the programs and, less often, in the analytical evaluation. Such errors must be corrected to restore trust in the results obtained. Third, while individual corporations often have a fondness for particular economic risk programs, one should not allow such fondness to override the evaluation process. One should, at the least, evaluate the estimated economic risk using programs based on different risking methods to ascertain the commonality and/or differences in the assessments so that one obtains some idea 
of the relevant uncertainty attendant upon using a favorite program.

There is a considerable body of literature available concerning economic risking methods in hydrocarbon exploration so that one can legitimately ask: why does one need a review? The answer is that in much of the literature, one can question whether proper treatment of basic assumptions, analytic versus numerical solutions, or comparison of different risking methods has been properly addressed. In addition, much of what is known about different risking methods is scattered throughout the literature in many different publications in many different journals so that a review is an appropriate place to collect in one place the basic outlines of such methods. There is also the point that recent investigations have often uncovered curious results that at first sight seem not only strange, but also incorrect and that after considerably more in-depth understanding can be shown to remain either curious but correct or curious and incorrect. Blind trust in a numerical program will not usually enable one to see such curiosities or flaws in basic procedures. Hence the need for a review article.

References are not given in the text unless crucial to the review, but a sampling of available articles and/or books dealing with economic risk evaluation in hydrocarbon exploration is provided at the end of the review. The reader can extend the list given almost indefinitely by undertaking an Internet search.

Finally, before proceeding with quantitative examples, it is appropriate to note that much of the detailed mathematical development for the different risking methods discussed here has been relegated to several appendices. This relegation has been done so that the main body of the review is not cluttered with each and every mathematical development that could be off-put to the average reader. In this way, one can present the main results and underlying basic assumptions in a fashion that can be understood by most readers. Those who are interested in mastering the detailed quantitative methodology can consult the appropriate appendices.

\section{Introduction}

There exists a large number of quantitative methods for assessing financial risk and corresponding involvement in hydrocarbon exploration projects to optimize the level of diversification. However, the resulting assessments of each such method are often very different or even in conflict so that one is unsure which (if any) of the methods provides an evaluation that is trustworthy.

In addition, some procedures, such as the Cozzolino (1977) formula for determining optimum working interest in a project, indicate that involvement in potentially very attractive projects should be avoided or minimized-in complete contrast to that which one would consider as appropriate. This point is addressed quantitatively in "Appendix 1" for the Cozzolino risking procedure, although it is equally valid for all utility-based risking methods. As a consequence of such unacceptable behavior and the complexity of the calculations together with the need to provide an assigned risk tolerance, the Cozzolino method has lost much of its previous luster as an acceptable instrument for evaluating financial risk and diversification in hydrocarbon exploration.

In the search for other risking methods that indicate one should invest in attractive projects (to the maximum extent concomitant on not jeopardizing a corporation's financial health through gambler's ruin), there has been progress made in utilizing methods based on direct probability without the appearance of the exponential behavior that is of fundamental importance in the Cozzolino procedure (see "Appendix 1"). Two such different risking methods are examined here: one usually named the Kelly method and the other related to corporate risk probability. As will be shown, both have their limitations and so the search for a stable and robust risk procedure is still a matter of extreme interest. Consider each of the two risking methods in turn.

\section{The Kelly risking method versus the Cozzolino risking method}

\section{Theoretical considerations}

In a seminal paper, Kelly (1956) argued that one could quantify the financial risk associated with a project in terms of potential losses and potential gains and the probability that each would occur. (Actually, Bernoulli 1738 had advanced a similar argument to that of Kelly, but Bernoulli's work was not available in English until 2 years before Kelly did his work and it is doubtful whether Kelly was aware of Bernoulli's prior contribution.) The basic scheme calculates the fraction of one's available capital that should be spent on a project. In the simplest form: if the values of success and failure are the same, then the fraction is simply the difference between the probability of success and the probability of failure. For instance, let the present success value be $\$ 10$, the present failure value also be $\$ 10$, and set the chance of success at $70 \%$. Then the Kelly fraction is just $70 \%-30 \%=40 \%$ so that $40 \%$ of one's available capital up to a maximum of $\$ 10$ should be invested. Since 1956, a considerable number of articles have explored the use of the Kelly risking method mainly in the fields of gambling strategies and stock market investments, where one can undertake a large number of games and/or investments at once or in series. A small set of relevant references for such fields is presented in the reference list at the end of this paper. While 
the main ideas in that set of references are of interest, they are not quite as useful in the field of hydrocarbon exploration as they are in the fields of gambling and/or stock market investment because one rarely gets the chance to set up a large number of games or investments with unlimited investment potential in each for hydrocarbon exploration strategies. Instead, one has to be more concerned with the chance of a relatively small number of wells or hydrocarbon plays returning a profit. One is also limited to the total well cost of $100 \%$ and so one cannot take, say, $500 \%$ in a very good project. In contrast, one can buy as many shares as one wishes on the stock market or $\$ 100$ bets on a horse to win a race. It is this difference that makes the hydrocarbon exploration risk somewhat different from the financial risk of a large number of investments. One also does not usually have the luxury of taking gains and keeping them for another opportunity - instead, such gains are mostly returned to the corporate coffers and one must deal anew with the corporate budget makers for the following year. For these reasons, it is appropriate to adapt the Kelly risking method to the hydrocarbon exploration theater.

The concern in this paper is with the three commonly used methods: the Cozzolino risk method, the Kelly risk method and the corporate confidence risk method. One already knows there are disturbing aspects to the Cozzolino approach as outlined in "Appendix 1" that makes the method less trustworthy than one might have anticipated at first glance, so less emphasis will be placed on that method in the body of the paper.

The fundamental difference between the Cozzolino approach and the Kelly method runs roughly as follows, and written in terms of hydrocarbon exploration parameters the arguments can be expressed as the net cash value (NCV) in terms of the costs, $C$, the estimated gains, $G$, the success probability, $p$, the available investment capital, $B$, for the individual project and not the total available capital the corporation has on hand - usually much in excess of the amount one should spend on an individual project that, in turn, is limited to the costs $C$, and the risk tolerance RT, all of which are estimates prior to actual drilling. The net present value (NPV) is usually considered to be $G-C$. Cozzolino provided a risk method that basically argued the one should consider maximizing the logarithm of the sum of two terms: the first being the success probability multiplied by a nonlinear function, $F$, of the gains minus costs should one be successful, i.e., $p_{\mathrm{s}} F(G-C)$, and Cozzolino chose $F(G-C)$ to be $\exp (-(G-C) W / \mathrm{RT})$ where $W$ is the working interest one should take in the project; the second factor is the failure chance $\left(1-p_{\mathrm{s}}\right)$ multiplied by the same nonlinear function $F$, but now involving only the costs, i.e., $\left(1-p_{\mathrm{s}}\right) \exp (W C / \mathrm{RT})$, so that the total logarithm to be maximized is of the form $\ln \left(p_{\mathrm{s}} F(G-C)+\left(1-p_{\mathrm{s}}\right)\right.$ $F(-C)$ ). Appendix 1 discusses in detail the maximization of the Cozzolino method (there is also a basic question as to why one should choose an exponential behavior at all as opposed to any other mathematical structure). Table 1 provides four examples of project assessment using the Cozzolino method when the available capital for each project is the same as the risk tolerance $(B=\mathrm{RT})$.

In contrast, Kelly chose to maximize the sum of two logarithms taken together but each multiplied by either $p_{\mathrm{s}}$ or by $\left(1-p_{\mathrm{s}}\right)$, respectively, and with each entry in the respective logarithms being linear in the form either $1+(G-C) / B$ for the logarithm factored by $\mathrm{p}$ or $(1-C / B)$ for the logarithm factored by $(1-p)$, so that the total factor to be maximized can be written as $\mathrm{p}^{*} \ln (1+(G-C) / B)+(1-p) \ln (1-C / B)$. The purpose here is not to recommend either of the risk methods, but rather to accept them "as is" and then to ask what are the consequences of each for risk evaluation of a project.

In the case of the Cozzolino scheme, there have been significant investigations of its strengths and weaknesses (see e.g., Lerche and MacKay 1999 for a very detailed treatment) and "Appendix 1" provides a short précis of the main drawbacks of the Cozzolino method.

The emphasis then in this section will be on the Kelly prescription with detailed mathematical developments given in "Appendix 2". An analogy helps: consider the Kelly fraction as the optimum trajectory for a cannon to shoot a projectile the maximum distance under gravity. If the firing angle is too low, the projectile falls short of the maximum distance, while if the angle is too high then again the projectile falls short. Only one angle achieves the maximum distance. For the Kelly criterion, the corresponding statement is that only a single fraction of one's investment capital achieves the maximum long-term return on investment.

Basically for a given investment per project, $B$, one obtains from "Appendix 2", (Eq. 13), the optimum working interest, $f_{\text {opt }}$, one should take in the project as

$f_{\text {opt }}=(B / C) F_{\max }$,

while the maximum fraction that optimizes the Kelly scheme in terms of the highest long-term geometric rate of return is (MacKay 2015)

$F_{\max }=(p G-C) /(G-C)=\mathrm{EV} / \mathrm{NPV}$

Table 1 Four projects evaluated using the Cozzolino method. OWI refers to the optimum working interest one should take in a project using (Eq. 9) of Appendix

\begin{tabular}{lllll}
\hline Projects & $A$ & $B$ & $C$ & $D$ \\
\hline Cost $(C)$ & $\$ 4$ & $\$ 3$ & $\$ 2$ & $\$ 1$ \\
Gain $(G)$ & $\$ 5$ & $\$ 10$ & $\$ 20$ & $\$ 50$ \\
Chance $(p)$ & $100 \%$ & $50 \%$ & $25 \%$ & $10 \%$ \\
Available capital $(B=\mathrm{RT})$ & $\$ 5$ & $\$ 5$ & $\$ 5$ & $\$ 5$ \\
Expected value $\left(\mathrm{EV}=p^{*} G-C\right)$ & $\$ 1$ & $\$ 2$ & $\$ 3$ & $\$ 4$ \\
Cozzolino OWI $(\mathrm{COWI})$ & $100 \%$ & $42 \%$ & $27 \%$ & $17 \%$ \\
\hline
\end{tabular}


with the expected value as $\mathrm{EV}=p G-C$ and NPV $=$ $G-C$.

Table 2 repeats the structure of Table 1 for four different projects, but with the addition of the Kelly fraction $F_{\text {max }}$ and the optimum working interest $f_{\text {opt }}$ from the Kelly method. All working interest calculations are limited to the range 0-100\% inclusive. In Eq. (1), one must limit $f_{\text {opt }}$ to less than or equal to unity because one cannot take more than $100 \%$ involvement in a project.

Note in particular that as the gains increase without limit, the optimum working fraction $F_{\max }$ that one should take (Eq. 2) systematically increases to the value $p$, the success chance. However, the Cozzolino OWI decreases to zero at high gains, which causes one to be concerned about the correctness of the Cozzolino formula for OWI at the very least at high gains and perhaps also at lower gains. For the Kelly method to calculate the Kelly working interest, one must convert the Kelly fraction of the available capital to the working interest for the costs, i.e., (Kelly fraction) $* B=$ (Kelly working interest)*C. For the case of project $\mathrm{E}$ given in Table 2, the calculation is $17 \% * \$ 5=$ (Kelly working interest)*\$2, yielding a Kelly working interest of $42.25 \%$ which, when rounded, gives the result $42 \%$ as shown in the last row of column $\mathrm{E}$ in Table 2.

From the Kelly geometric framework, one can also provide the corresponding maximum anticipated NCV $\left(\mathrm{NCV}_{\max }\right)$, as shown in "Appendix 2", as

$\mathrm{NCV}_{\text {max }} / B=(1+\mathrm{EV} / C)^{\mathrm{p}}(1-p)^{(1-p)} /(1-C / G)^{(1-p)}$,

using Eq. (1) in the general formula (“Appendix 2", Eq. 14) for NCV.

Note that both $f_{\text {opt }}$ and $\mathrm{NCV}_{\max }$ are linear in the investment made and are otherwise dependent only on parameters related to the project values $(G, C, p)$. Thus how much one invests changes only the fractional involvement in a linear manner.

Table 2 Cozzolino and Kelly optimum working interest in four projects

\begin{tabular}{lllll}
\hline & $E$ & $F$ & $G$ & $H$ \\
\hline Cost $(C)$ & $\$ 2$ & $\$ 2$ & $\$ 2$ & $\$ 2$ \\
Gain $(G)$ & $\$ 20$ & $\$ 200$ & $\$ 2,000$ & $\$ 20,000$ \\
Chance $(p)$ & $25 \%$ & $25 \%$ & $25 \%$ & $25 \%$ \\
Available capital $(B=\mathrm{RT})$ & $\$ 5$ & $\$ 5$ & $\$ 5$ & $\$ 5$ \\
Expected value $\left(\mathrm{EV}=p^{*} G-C\right)$ & $\$ 3$ & $\$ 48$ & $\$ 498$ & $\$ 4,998$ \\
Cozzolino optimum working interest & $27 \%$ & $9 \%$ & $1 \%$ & $0 \%$ \\
$\quad($ OWI $)$ & & & & \\
Present value $(G-C)$ & $\$ 18$ & $\$ 198$ & $\$ 1,998$ & $\$ 19,998$ \\
Kelly fraction $(K=\mathrm{EV} / \mathrm{PV})$ & $17 \%$ & $24 \%$ & $25 \%$ & $25 \%$ \\
Kelly working interest & $42 \%$ & $61 \%$ & $62 \%$ & $62 \%$ \\
$\quad\left(\mathrm{KWI}=K^{*} B / C\right)$ & & & & \\
\hline
\end{tabular}

The main point of the Kelly scheme is to supply an OWI that does not depend on determining a risk tolerance in any form, but is solely dependent on parameters of the project and the investment capital available. The Kelly procedure achieves this goal with the maximum fraction OWI less than unity and simply expressed without any complex logarithmic factors but with the restriction EV must be positive.

In terms of application to hydrocarbon exploration projects, there are, nevertheless, several weaknesses with the Kelly scheme. First, note that both the Kelly method and the Cozzolino method maximize the average and therefore require a large number of projects to approach the objective results. This requires a significant amount of time if one drills only a few wells per year. Because both methods depend on the EV value, both require a positive EV to be applicable. Both the Kelly method and the Cozzolino method calculate a negative fraction if the $\mathrm{EV}$ is negative and are used in the stock market to indicate a preference for a short position (sell now and buy later).

By contrast, in hydrocarbon exploration, the trust one should place in such long-term averages is hard to justify for an individual exploration project that will be either a success or a failure so that the outcomes are either $G-C$ or $-C$. A multiplicity of attempts through long-term diversified portfolios is all one can predict. One cannot place any trust in short-term predictions with the phrase "This project looks extremely good and so it will work" because neither method is based on such individual scenarios. There are no other options (excluding the chance of a catastrophe).

Second, the Kelly scheme has the maximum NCV (see Eq. 3) positive always and never zero so that one is profitable (or at least not a loss of all available capital so that after a project is completed one will have some money left overassuming that is any consolation). This is in contrast to real exploration situations where one can (more often than one wishes) end up with a loss.

Third, note that the Kelly method as given here does not take into account uncertainty around the mean value. It is in contrast to simple EV calculations where one can easily show (Lerche and McKay 1999) that the standard deviation around the EV is given through the square root of the variance $V=G p(1-p)$. Some procedure for including an uncertainty around EV should be incorporated automatically, but runs the risk of yielding an EV distribution that includes negative values. One method for including variance is to track the EV, the variance of projects and the portfolio of all the projects. Both the Kelly method and the Cozzolino method react to changes in project variance and so allow one to assess the stability of the results (commonly measured by $\mathrm{EV} / \mathrm{V}^{1 / 2}$ ). A high value of $\mathrm{EV} / \mathrm{V}^{1 / 2}$ indicates a low uncertainty on an outcome and, conversely, a low value of EV/ $V^{1 / 2}$ would suggest significant uncertainty. Despite its shortcomings, the Kelly procedure has less unacceptable 


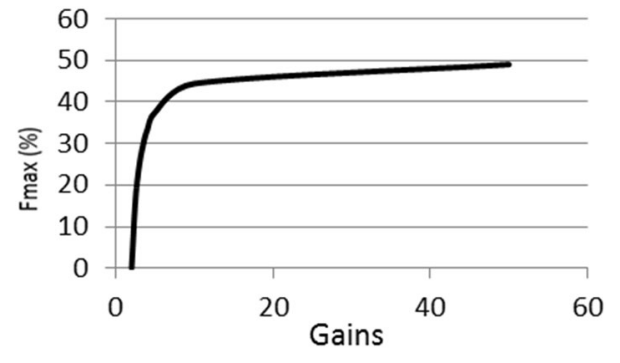

Fig. 1 Plot of $F_{\max }$ versus gains for the case $p=50 \%, C=1, B=1$. Note that $F_{\max }$ tends to the limit of $\mathrm{p}(=50 \%)$ as the gains rise indefinitely

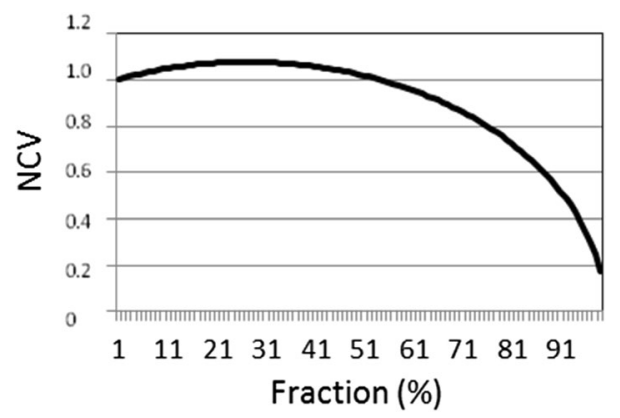

Fig. 2 Net cash value as a function of increasing fractional involvement for the case described in Fig. 1. One has an optimum working interest as shown in $27.7 \%$ and a peak NCV of 1.079

features than does the Cozzolino method and so provides a greater degree of trust when attempting to evaluate the risk of involvement in a hydrocarbon exploration project.

\section{A numerical example}

Consider the simple situation where $p=50 \%, C=1, B=1$ and the gain increases steadily from the minimum allowed value of $C / p=2$. A plot of $F_{\max }$ versus $G$ is given in Fig. 1 .

Figure 2 shows the net cash value as a function of increasing fraction for the situation $p=50 \%, C=1, B=1$ with gains $G$ set at 3.2 showing a maximum working interest of $27.7 \%$ when the NCV peaks at 1.079. For comparison, Fig. 3 plots the OWI from the Cozzolino formula showing the unacceptable behavior of a decreasing involvement as the gains rise systematically after reaching a peak value for OWI. Figure 4 plots RAV from the Cozzolino formula ("Appendix 1", Eq. 8) versus gains for the case $p=50 \%, C=1, \mathrm{RT}=1$.

For the Kelly situation if one were to consider the case where one does not have enough money to invest at the rate to cover all project costs, then both the working interest one can take in the project and the NCV are lowered. Fortunately, as shown above, the fraction of the project one should take is then simply given by $f_{\mathrm{opt}}=(B / C) F_{\max }$, which is just a

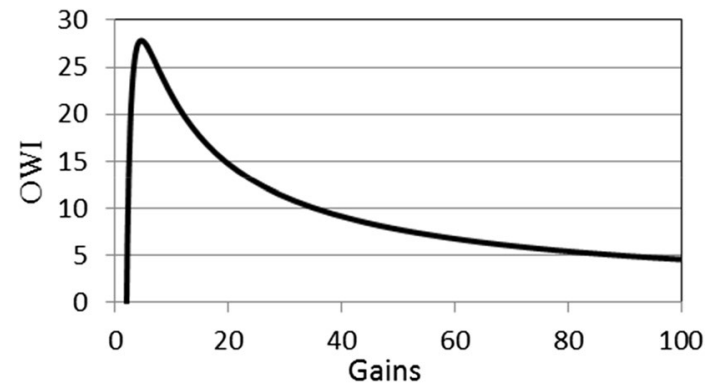

Fig. 3 Plot of OWI (in \%) from the Cozzolino formula ("Appendix 1", Eq. 9) versus gains for the case $p=50 \%, C=1$, RT $=1$. Note how the OWI decreases at high gains

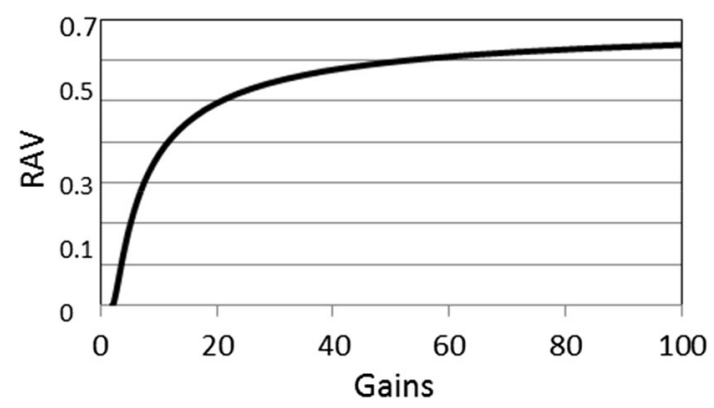

Fig. 4 Plot of RAV from the Cozzolino formula ("Appendix 1", Eq. 8) versus gains for the case $p=50 \%, C=1$, RT $=1$

linear proportion of the optimum Kelly fraction. Equally, the $\mathrm{NCV}$ is then also just the same fraction of the NCV appropriate for the situation where one can cover costs.

\section{Risking multiple projects with the Kelly method}

Despite its limitations as discussed above, the Kelly procedure has less flaws than the Cozzolino risking procedure and so likely provides a better assessment of risk of incorrect diversification decisions. For an individual project with $\mathrm{EV}>0$, there is no difficulty in determining the OWI. However, complications ensue when one has to deal with multiple projects. Suppose one is interested in $N$ projects, each with its own values for $G, C$, and $p$, but with each having a positive EV so that one can use the Kelly method. Two limiting considerations illustrate the problem: first (and the less likely case) the projects are to be considered in a time sequence so that after one project has been evaluated for its NCV the next in line is also to be evaluated and so on; second (the more likely case), the $N$ projects are to be considered simultaneously because the commitment for involvement in each is to be undertaken when the budget 
is approved-normally, around 6 months to a year before commencement of activities. Consider each scenario in turn.

\section{Sequential project evaluation}

Start with a total budget $B$. After the end of the first project, the NCV from the project is provided through the total amount $\mathrm{NCV}_{1}$ available. Relabel this amount as $B_{1}$ representing the increase (decrease) in the original budget.

Then, analyze the second project using $B_{1}$ as the available budget yielding an NCV after the second project of $\mathrm{NCV}_{2}$ which in turn is relabeled $B_{2}$ and is used to evaluate the next project and so on. In this way, one has parlayed the original budget (stake) sequentially so that after $N$ projects one has an amount $\mathrm{NCV}_{N}$. The assumption here is that one does not have a sufficient decline in the sequential NCV so that one cannot invest in some of the later projects. As such, this procedure has a similarity to betting on horse races at a track with each race following the next and winnings from each race are parlayed onto a bet for the next race. In short, one assumes from a hydrocarbon exploration prospective that there is complete reinvestment of capital in each sequential prospect-something that just does not occur in reality except for small corporations.
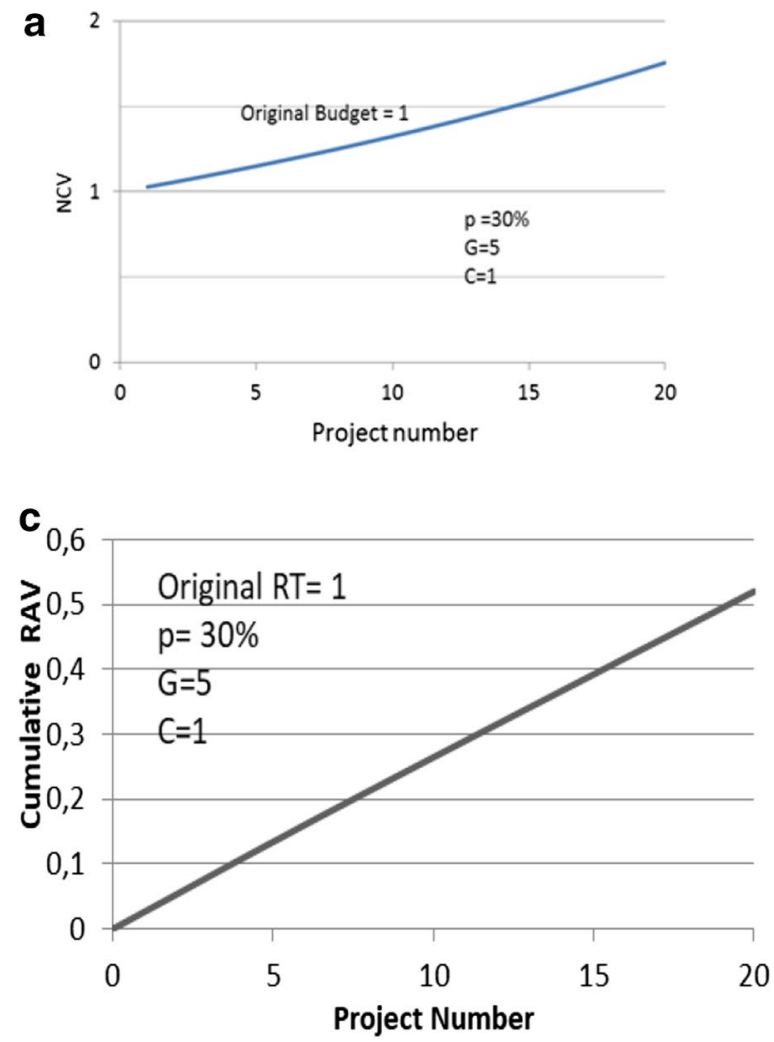

Figure 5 shows an illustration of this sequential evaluation for both the Kelly procedure (Fig. 5a, b) and the Cozzolino procedure (Fig. 5c, d) for success probabilities of $30 \%$ and $50 \%$ for both procedures.

\section{Simultaneous project evaluation}

When all projects are to be evaluated simultaneously, the assessment of the fractional involvement in each project is not as simple as the sequential situation. Fortunately, the basic method for effecting an evaluation has already been given (Lerche and MacKay 1999) for the Cozzolino procedure and requires only minor modification to be applied to the Kelly method, although the solution of some resulting equations would seem to be available only with numerical methods as shown in "Appendix 3". One can take the particular solution to the equations given in "Appendix 3" and then compute the fractional involvement in each project. In other words, one uses the optimum working interest for each project considered individually and then orders the involvement in proportion to the highest working interest (see Table 3). For any budget other than that given by Eq. (26) of "Appendix 3", the corresponding analytical solution of Eq. (25) is difficult to say the least. To keep matters
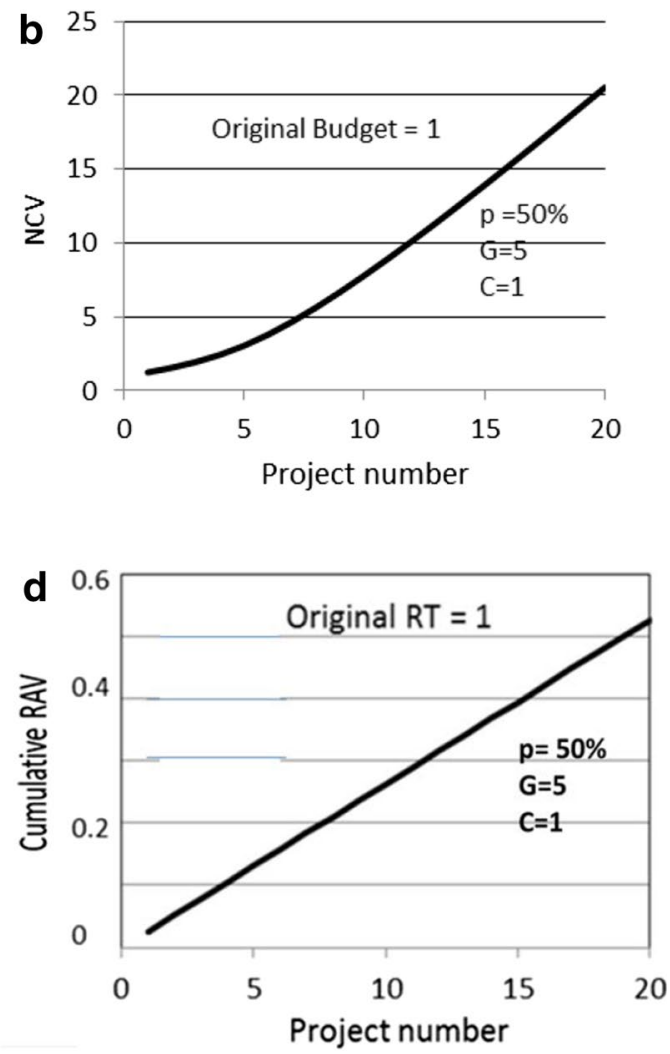

Fig. 5 Illustration of sequential evaluation for both the Kelly (Fig. 5a, b) and Cozzolino procedures (Fig. 5c, d) for success probabilities of 30\% and $50 \%$ for both 
Table 3 Kelly working interest for five projects considered simultaneously

\begin{tabular}{lrrlllllrr}
\hline EV/PV & \multicolumn{1}{l}{ PV } & EV & Project & $G$ & $C$ & $P$ & $B$ & Est. $f$ & Kelly WI \\
\hline $4.00 \%$ & $\$ 500.00$ & $\$ 20.00$ & 1 & 600 & 100 & 0.2 & 500 & $20.00 \%$ & $20.00 \%$ \\
$6.25 \%$ & $\$ 2,000.00$ & $\$ 125.00$ & 2 & 2500 & 500 & 0.25 & 500 & $6.20 \%$ & $6.20 \%$ \\
$2.86 \%$ & $\$ 700.00$ & $\$ 20.00$ & 3 & 800 & 100 & 0.15 & 500 & $14.29 \%$ & $14.29 \%$ \\
$68.00 \%$ & $\$ 50.00$ & $\$ 34.00$ & 4 & 80 & 30 & 0.8 & 500 & $100.00 \%$ & $100.00 \%$ \\
$5.18 \%$ & $\$ 140.00$ & $\$ 7.25$ & 5 & 265.5 & 125.5 & 0.5 & 500 & $20.63 \%$ & $20.63 \%$ \\
\hline
\end{tabular}

Table 4 Array of five projects all with the same expected value (EV)

\begin{tabular}{lrrll}
\hline Project number & Gain & Cost & $\begin{array}{l}\text { Prob. success } \\
(\%)\end{array}$ & EV \\
\hline 11 & 100 & 10 & 20 & 10 \\
22 & 50 & 5 & 30 & 10 \\
23 & 200 & 20 & 15 & 10 \\
44 & 75 & 15 & 33.3 & 10 \\
55 & 80 & 40 & 62.5 & 10 \\
\hline
\end{tabular}

simple, an alternative approach is to consider that one does not start with an arbitrary budget, but rather with a budget given precisely through Eq. (26) and then the solution for the various fractions of the budget to use for each project are indeed given through Eq. (27). As a practical matter, some might prefer this approach. Note also that the optimum fractions $\mathrm{f}_{\mathrm{k}}$ of the budget for each project with $\mathrm{EV}_{\mathrm{k}}>0$ are less than unity and greater than zero with the choice $H=0$ (see "Appendix 3") which is, at the least, encouraging, although not a complete resolution of the fractions to take when the available cash, $B$, is less than $\Sigma C_{\mathrm{i}}$. There is, fortunately, a numerical scheme available for other choices to solve Eq. (25) based on Excel Solver@ that permits one to set ranges for any and all parameters involved and then the program finds the values that yield the optimum fractions for any and all choices.

\section{Numerical example}

Consider the array of five projects listed below in Table 4 . The values of costs, gains and success probabilities have been chosen so that it is not obvious which project is better than the others, because they all have the same EV value as also listed in Table 4. Figure 6a shows the costs per project at Kelly OWI, while Fig. $6 \mathrm{~b}$ shows the Kelly OWI per project.

Applying the Kelly risking method for the case of optimum involvement (and so one must have available the sum of the costs for all five projects, i.e., 90) allows one to calculate the OWI for each of the projects. Shown in Fig. 7 are the OWI values (in \%). Based solely on OWI, one would then rank the projects in the order 5,2, 4, 1,3 indicating that just because there is the chance of high gains (as in project 3), the low probability of success downgrades the project and

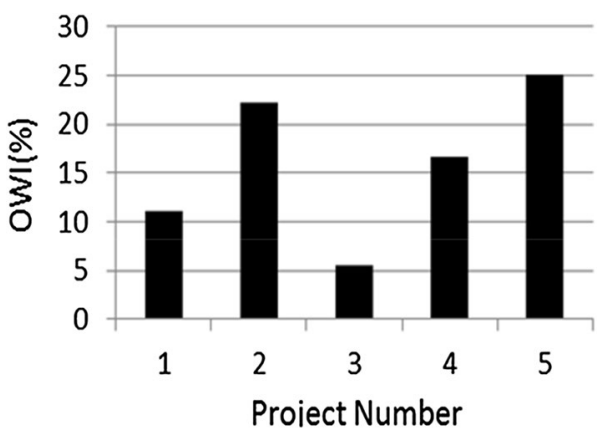

Fig. 7 OWI values (in \%) for each of the five projects listed in Table 4; all have the same EV
Fig. 6 a Costs per project at Kelly OWI; b Kelly OWI per project

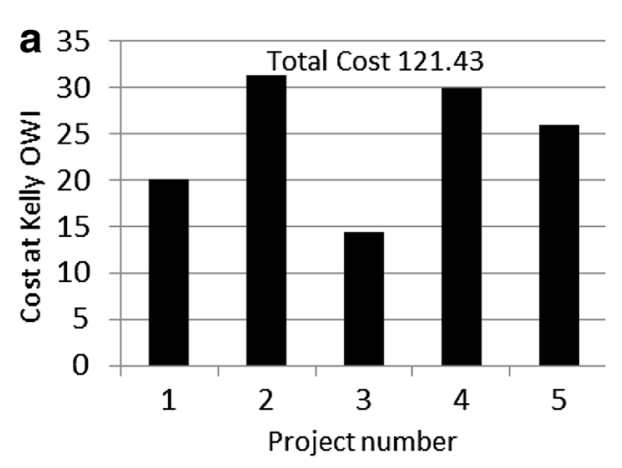

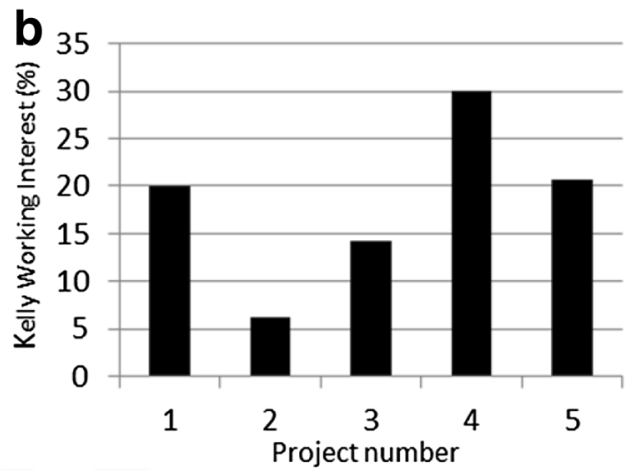

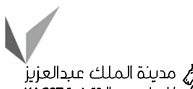

لرئر مدينة الملك عبدالعزيز Springer 
the low gain project 5 has a much better chance of yielding a positive result because it has a considerably better chance of success.

Of course, the ordering of the OWI values for the five projects does not imply the same NCV for each. Shown in Fig. 8 are the corresponding NCV values based on the Kelly geometric mean procedure. If one were to use the NCV as the sole decision criterion for investing, then one would rank the projects in the order 5, 3, 4, 1, 2 so that the estimated gains would then play a more significant role in assessing project worth than the OWI.

How one decides what to do is then a matter of the corporate acceptance of the risking method and its estimates based on different factors-in this case OWI versus NCV. Perhaps, the illustration is simple, but it is the very simplicity that enables one to see how the different factors influence results and that have been the main point of the example. An alternative prescription is based on corporate confidence and that method is discussed next.

\section{The corporate confidence method}

Dissatisfaction with the Cozzolino risking method has led to suggestions for risk assessment procedures that depend on the attitude of a corporation to risk tolerance. The rationale is as follows. The Cozzolino method for determining the optimum working interest (OWI) one should take in an exploration project is based on the underlying basic utility theory (see "Appendix 1"; Lerche and MacKay 1999; Miam 2002). As such, the method suffers from the generic consequences of all utility-based methods that: (i) the OWI is never guaranteed to be always less than unity as it must be; (ii) the OWI does not always increase as the net present value of a project is increased as it should; and (iii) the OWI is automatically set to zero when the expected value of the project is less than zero, despite there being an uncertainty on the expected value that could still allow a profitable

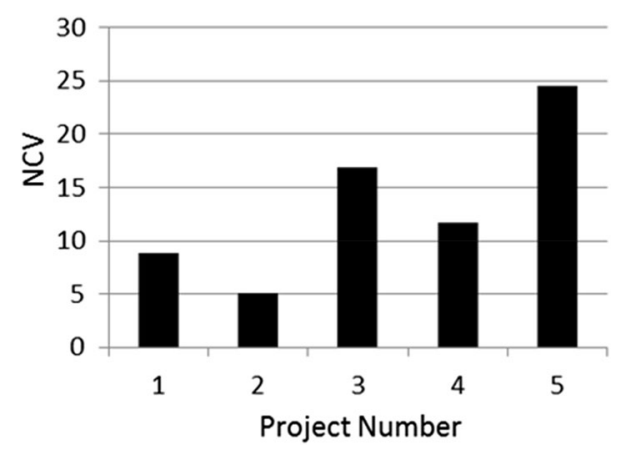

Fig. 8 NCV values for each of the five projects listed in Table 4; all have the same EV outcome. All utility-based risking methods have the same flaws. As a consequence of these drawbacks, here a binomial procedure is discussed for ascertaining the OWI, which does not suffer from any of the utility-based problems. In addition, one can also compute the corporate confidence in a project using this approach, and determine the corresponding apparent confidence of any utility-based procedure for OWI. This binomial procedure for determining OWI and corporate confidence is, therefore, a better alternative over previously available methods (Lerche and MacKay 2003).

There are two distinct procedures for obtaining a corporate confidence estimate of an exploration project. Consider each in turn.

\section{Risk estimates based solely on corporate risk tolerance}

Introduce a binomial corporate confidence probability, $p_{\mathrm{c}}$, representing the corporate confidence that at least one project from many similar projects will be a discovery with a corporate risk tolerance RT (measured in \$). To relate the confidence probability to an optimum working interest (OWI), one can write:

$p_{\mathrm{c}}=1-(1-p)^{a /(1-a)}$,

with $\mathrm{a}=\mathrm{OWI}(1+\mathrm{RT} / C)$ and where $p$ is the success probability. At the lowest value of $a=0$, one has, $p_{\mathrm{c}}=0$ indicating no confidence that the project will be successful, while at the largest value of $a=1$ one has $p_{\mathrm{c}}=1$ indicating $100 \%$ confidence the project will prove successful. One can invert Eq. (4) to obtain:

OWI $=(1+C / \mathrm{RT})^{-1} \ln \left(1-p_{\mathrm{c}}\right) /\left(\ln \left(1-p_{\mathrm{c}}\right)+\ln (1-p)\right)$.

Note that Eq. (5) always has OWI $<1$; indeed, $\mathrm{OWI}<(1+\mathrm{C} / \mathrm{RT})^{-1}$, so that one can choose to work with a corporate confidence and so determine the OWI or one can work with a given OWI and so estimate the corporate confidence. As an added bonus, one could insert the OWI from the Cozzolino formula (see "Appendix 1") into Eq. (5) and so determine the apparent corporate confidence of the OWI value. A better alternative is to use the Kelly risking method. Take the five projects given in Table 4 and use the individual OWI values to evaluate the corporate confidence probability for each project (for illustrative purposes, the value $\mathrm{RT}=\mathrm{C}$ has been chosen for each project). As shown in Fig. 9, the confidence probability one places on each project is a strong statement that one be either conservative or at worst neutral in investing in three of the five projects (numbers 1, 2, 4 in Fig. 9), because their corporate confidence probabilities are much less than $10 \%$. For project 2 , the confidence that it will be successful is just around the $10 \%$ mark, while project 5 , with the highest success chance (62.5\% given in Table 4$)$ 


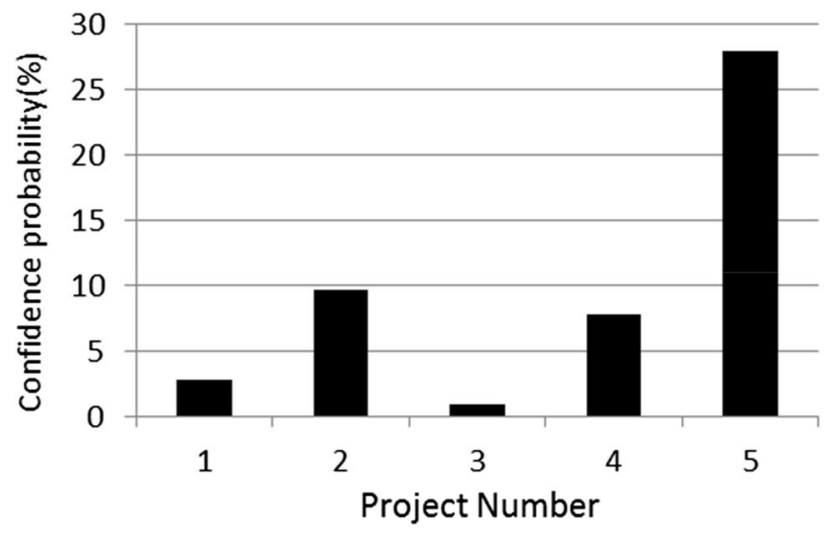

Fig. 9 Corporate confidence probability plotted against the project number for the case where the OWI is taken from the Kelly formalism for the five projects given in Table 4

although not with the largest possible gains, is favored in terms of the confidence one attaches to each project.

While Eq. (5) represents a distinct improvement on previous methods, it suffers from the drawback that the OWI is not dependent on the value of the project should it succeed (i.e., on $G-\mathrm{C}$ ), although the main aim of including some form of corporate confidence is achieved. To include the value of the project in an assessment of risk, one considers the extension of Eq. (5) with gains and costs included.

\section{Risk estimates based on project value}

Take Eq. (5), multiply the right hand side by $(1-C / G)$ and so define an OWI as

$$
\begin{aligned}
\text { OWI }= & (1-C / G)(1+C / \mathrm{RT})^{-1} \ln \left(1-p_{\mathrm{c}}\right) /\left(\ln \left(1-p_{\mathrm{c}}\right)\right. \\
& +\ln (1-p))
\end{aligned}
$$

with the corresponding inverse formula

$p_{\mathrm{c}}=1-(1-p)^{b /(1-b)}$,

where $b=a^{*} G /(G-C)$. In this case, the limits on OWI are $0<$ OWI $<(1-C / G)(1+C / \text { RT })^{-1}<1$.

This estimate is based on the value $(G-C)$ of a project and so permits higher OWI to be realized for higher gain projects or higher corporate confidence for higher gain projects. As shown in Fig. 10, the ordering of the five projects in terms of corporate confidence does not change, but the estimate of confidence one should place in each project is affected with almost $50 \%$ confidence for project 5 and over $10 \%$ for project 2 . The remaining three projects remain at confidence values of less than $10 \%$. In short, the implications are that it is better to take a higher corporate chance on a lower potential gain project (number 5) than on higher

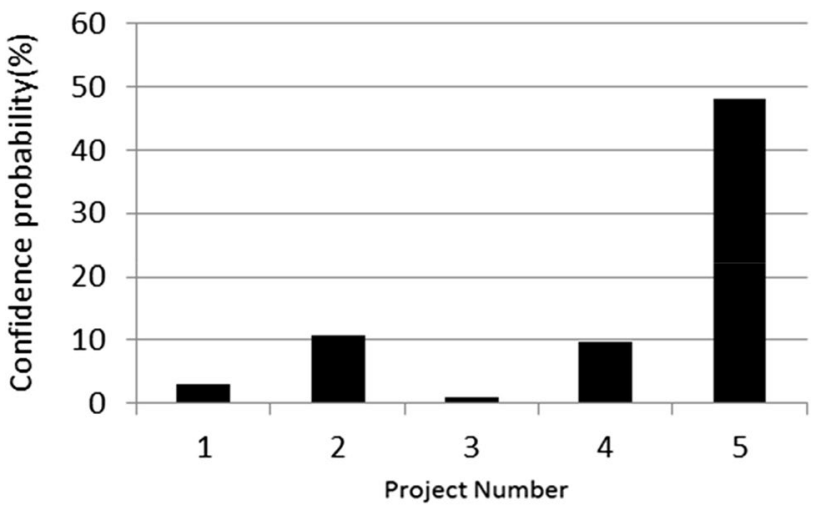

Fig. 10 Corporate confidence probability plotted against the project number for the case where the OWI is taken from the Kelly formalism for the five projects given in Table 4 and when the potential gain for each case is built into the estimate of corporate confidence

potential gains but a much lower chance of success (project 2 for instance).

The decision as to whether one should use Eq. (4) or Eq. (7) is then a matter of corporate strategy because Eq. (4) does not include any information about individual project worth and so treats high and low value projects equally, while Eq. (7) permits higher values for OWI for higher gain projects. Note also that both equations permit an assessment of NCV for any NCV $>0$ and not just for projects with EV $>0$; this is in marked contrast to both the Cozzolino procedure and the Kelly procedure. Note further that both corporate confidence methods indicate that projects with a high failure chance (lowered values of $p$ ) will have a lower value of $p_{\mathrm{c}}$, while projects with a higher chance of success will have correspondingly higher values of corporate confidence probability. Such information is precisely that required by a corporation to decide on investment in a particular project in comparison to other projects.

While the two corporate confidence risking methods have notable advantages over utility-based methods and also over the Kelly method (perhaps, the most obvious advantage being that one can calculate the risk when $\mathrm{EV}<0$ - something that is not possible with utility-based methods or the Kelly prescription), there are still some drawbacks in corporate confidence risking methods. First, one has to introduce another parameter (corporate confidence probability) to perform an acceptable risk evaluation. In and of itself, such is not particularly damaging because the limitations of the utility and Kelly methods are then overcome immediately and several positive aspects ensue as shown above. However, one would prefer to have a method of assessing risk that did not need to introduce "extra" parameters. Second, there is the unsettling fact that one considers the OWI with arbitrary (but linear) multiplicative factors such as $(1-C / G)$ and $(1+C / \mathrm{RT})^{-1}$. There is no obviously compelling basis for 
such choices - one could equally multiply by any equivalent nonlinear factors, for example $(1-C / G)^{3}$ and $(1+C / \mathrm{RT})^{-3}$ or any other values. There seems to be no formal basis available to determine what multiplicative factors are the best to choose or, indeed, what constitutes "best" - except for the pragmatic fact that the linear choices do show a procedure that provides an acceptable resolution to the problems associated with the utility and Kelly methods. Presumably so do many other choices, so how one goes about sorting out the most appropriate factors to use is still an open question.

\section{Discussion and conclusion}

Involvement in hydrocarbon exploration is a very expensive endeavor. For this reason, methods that attempt to minimize the risk associated ahead of actual drilling are considered of major importance in the oil corporate world to try to minimize cost and maximize gains. As shown here, the three cases of the Cozzolino risk method, the Kelly risk method and the corporate confidence risk method have advantages and disadvantages. In the case of the Cozzolino method, the disadvantages outweigh by far any advantages and so the use of this method has fallen into considerable disfavor over the years. A discussion of serious drawbacks of the Cozzolino method is given in "Appendix 1" and in more detail in Lerche and MacKay (1999, 2003). Therefore, preference for a more trustworthy method of assessing risk in hydrocarbon exploration has led to consideration of the Kelly method as a suitable alternative.

As shown in the text and discussed in detail in "Appendix 2", the Kelly prescription allows one to estimate the OWI only when one is dealing with situations where the expected value is positive, and also is based on the assumption one has a long run of situations-something that may be appropriate in the gambling and stock market worlds but is extremely unlikely to happen in the hydrocarbon exploration world! The Kelly prescription does, however, overcome many of the ills associated with the Cozzolino approach and so has been greeted with greater enthusiasm and the calculation of the OWI is far easier than that for the Cozzolino approach.

On the other hand, the ability to describe hydrocarbon exploration risk only when the expected value (EV) is positive is a bit of a drawback for there are situations where the EV can be negative but one still has a good chance of a positive outcome. The Kelly method would not allow one to invest when the $\mathrm{EV}$ is even one cent negative and one already knows there is an uncertainty on the EV through the standard deviation that indicates one can indeed have negative values. A way around this problem is given in "Appendix 4".

In attempts to overcome this hurdle, there has been investigation of a corporate confidence method (Lerche and MacKay 2003) that allows a corporation to provide what the corporation deems an acceptable confidence level in the OWI.
While the method solves almost all the problems associated with the Cozzolino and Kelly procedures, there is nevertheless a concern that there is a somewhat ad hoc development of relevant factors to choose to enable a corporation to evaluate the risk of involvement in a hydrocarbon exploration project. The search for a better method of risking continues.

Acknowledgements I am grateful to Matthias Biedermann and Jim McKay for their comments and without whose comments the work would, most probably, never have been finished.

Open Access This article is distributed under the terms of the Creative Commons Attribution 4.0 International License (http://creativeco mmons.org/licenses/by/4.0/), which permits unrestricted use, distribution, and reproduction in any medium, provided you give appropriate credit to the original author(s) and the source, provide a link to the Creative Commons license, and indicate if changes were made.

\section{Appendix 1}

The Cozzolino utility method.

The basic Cozzolino (1977) utility risking procedure operates using the formula:

$\mathrm{RAV}=-\mathrm{RT} \ln (p \exp (-W(G-C) / \mathrm{RT})+(1-p) \exp (\mathrm{WC} / \mathrm{RT}))$,

where RAV is the risk-adjusted value of the project, RT is the corporate risk tolerance, $W$ is the working interest to take in the project, $p$ is the probability the project will succeed, and $G$ and $C$ are the gains and costs, respectively.

To determine the optimum working interest (OWI) a corporation should take in the project, one differentiate (Eq. 8) with respect to $W$ and set the derivative to zero. This procedure produces the OWI given analytically through

OWI $=(\mathrm{RT} / G) \ln (p(G-C) / C(1-p))$.

When this OWI is substituted into Eq. (8), the corresponding RAV is given through

$\operatorname{RAV}_{\max }=(C / G) \ln (p(G-C) /(C(1-p))-\ln (1-\mathrm{EV} /(G-C)))$.

However, this OWI has some very unsavory features accounting for the reason why the use of the Cozzolino method has fallen into disfavor over the last 20 years or so as an appropriate risking procedure (and indeed all utility based methods suffer from similar shortcomings). Note first that projects with high gains are, from Eq. (9), to be invested in with smaller and smaller OWI-something that makes no sense at all. One requires that a high involvement should be undertaken in high gain prospects. Note secondly that there is no constraint on OWI from Eq. (9), while, because one cannot take more than $100 \%$ interest in a project, one must have OWI $<1$ everywhere for all situations (and such is the case only when $G>C+\mathrm{RT}$ ). The third disturbing factor is that the OWI of 
Eq. (9) is zero when $p G-C=0$, i.e., an $\mathrm{EV}=0$. For $\mathrm{EV}<0$ the Cozzolino formula for OWI does not allow an OWI that is real-the implication is that for $\mathrm{EV}<0$ one should not invest in the project. One suggestion for handling the problem of the Cozzolino formula yielding lower and lower OWI values as the gains increase has been to find the maximum OWI for lower gain values and to replace the OWI at high gains by this lower gain OWI value; the maximum OWI occurs at RT/ $(G-C)$ when $G=C(1+x)$, where $x$ satisfies $x=(1-p) \exp ((1+1 / x) / p)$. Such a procedure is less than satisfactory because essentially it is purely ad hoc, says that the actual gains estimate of a project are unimportant, and arbitrarily chooses an OWI that has nothing to do with the determination based on the Cozzolino formula - which is wrong anyway. There is no good reason to suppose that such an ad hoc procedure has any validity.

An alternative point of view is to demand that the OWI remains less than unity under all conditions. In that case, define

$\mathrm{RT}_{\max }=G / \ln (\mathrm{p}(G-C) / C(1-p))$,

so that one has

$\mathrm{OWI}=\mathrm{RT} / \mathrm{RT}_{\max }$.

One sees immediately that to hold OWI at a fixed value as the gains increase without limit, one must have $\mathrm{RT}=\mathrm{OWIxRT}_{\max }$ so that the corporate risk tolerance must rise in proportion. This behavior is unacceptable to a corporation.

It is these fatal flaws with the Cozzolino method that have led to the abandonment of utility-based procedures and have turned the focus to methods based more on binomial conditions. The Kelly method is one such procedure (see main text) and, while it successfully handles the first two drawbacks of the Cozzolino scheme, it, too, does not permit investment in a project when $\mathrm{EV}<0$.

\section{Appendix 2}

The Kelly geometric mean method.

The Kelly prescription can be written in the non-logarithmic form

$\mathrm{NCV}=B(1+f(G-C) / B)^{p}(1-f C / B)^{(1-p)}$,

where $f$ is the fraction of the available investment $B$ that should be used for the project and one has the constraint $B<C$. Note for further use that $1 \geq f \geq 0$. Note also from Eq. (13) that if one does not invest in a project $(f=0)$, then $\mathrm{NCV}=B$ so one retains one's money. If one invests the total available investment $(f=1)$ in a project with zero gains $(G=0)$, then NCV $=B-\mathrm{C}<0$ so that one lowers the available cash thereafter for the costs of the project. The increase (or decrease) in the cash after investment is then given by
$\mathrm{DNPV}=\mathrm{NCV}-B$

and measures directly the expected gain or loss of the project depending on the fraction $f$. One wishes to maximize the worth of the project and so one searches for the fraction that maximizes NCV.

Simple differentiation of NCV with respect to $f$ and then setting the derivative to zero provide the value of $f$ that maximizes NCV. This value is

$f_{\max }=B * \mathrm{EV} /(C(G-C)) \equiv B / B_{\max }$,

where $\quad \mathrm{EV}=p G-C$ and $B_{\max }=\mathrm{C}(G-C) / \mathrm{EV} \equiv$ $C(G-C) /(p G-C)$, and provides the maximum NCV of

$\begin{aligned} \mathrm{NCV}_{\text {max }}= & B(1+\mathrm{EV} / C)^{p}(1-\mathrm{EV} /(G-C))^{(1-p)} \\ & \text { when } B<\min \left(C, B_{\text {max }}\right)=B * \text { with } 1 \geqslant f_{\text {max }} .\end{aligned}$

Note in particular, the restriction $B<C$ must also be in place. In this latter event, one invests just $B^{*}$ in the project and so has $B-B^{*}$ left over for other projects. Note that one must have $B_{\max }>0$ to invest and so only those projects with $\mathrm{EV}>0$ (i.e., $G>C / p$ ) are to be considered using the Kelly risk formalism. In addition, note that because $B<C$ then one has $f_{\max }=(B / C) F_{\max }$ with $F_{\max }=(p G-C) /(G-C)$. Thus, the absolute best investment to make (the optimum working interest, OWI) based on the Kelly method is $F_{\max }$, but if one does not have enough money, $B$, to cover the total costs $C$ then one can only take a piece of the action available given through $f_{\max }$.

Consider now $\mathrm{NCV}_{\max }$. One is interested in just those projects where $\mathrm{NCV}_{\text {max }}$ exceeds $B$ (in the case that $B<B^{*}$ ). Thus for $B<B^{*}$, one considers solely those projects for which

$\operatorname{pln}(1+\mathrm{EV} / B)+(1-p) \ln (1-\mathrm{EV} /(G-C))>0$.

Some of the limitations of the Kelly method are as follows. First, one cannot handle situations where $\mathrm{EV}<0$; second, if one is interested in obtaining an NCV greater than the invested amount, then one has the restrictions on projects as expressed through (Eq. 17). However, the uncertainty on the $\mathrm{EV}$ is given by the variance $\sigma^{2}=p(1-p) G^{2}$ so that at the $1 \sigma$ level it is certain that EV $-\sigma$ will be negative for $p<50 \%$ and, more generally, will be negative whenever $G\left(p-\left(p(1-p)^{1 / 2}\right)\right)<C$. Such projects are still possible investment opportunities. An evaluation is not possible with the Kelly method because of the limitation to situations with the estimated EV $>0$.

On the positive side, the Kelly method does limit the amount (to $B^{*}$ ) one should invest in a project and also provides an optimum working interest one should take in a project (for $B<B^{*}$ ) through $f=B / B_{\max }$ with the restriction $B<C$. For very high gain situations, the ultimate OWI one should take is $F_{\max }=p_{\mathrm{s}}$. Note that when $\mathrm{EV}=0$, the value of $B_{\max }$ is infinite so that any finite available amount would 
imply the investment fraction should be zero. Accordingly while the Kelly method provides a significant improvement over the Cozzolino risking method (that in particular argues for less and less involvement in high gain situations-see "Appendix 1"), there are still drawbacks to its use although the situations where these drawbacks make their presence felt are not likely to be for projects where one would wish to invest too much money in general anyway.

\section{Appendix 3}

Multiple projects with the Kelly risking method

Consider $N$ projects but requiring now simultaneous evaluation for the fractional involvement to be undertaken in each. Let the $i$ th project have the values $\left(G_{i}, C_{i}, p_{i}, f_{i}\right)$ and an investment amount $B_{i}\left(<C_{i}\right)$ so that the total investment is $B=\Sigma B_{i}$. The requirement that the investment be spread among the $N$ projects requires that

$\Sigma f_{i}=1$,

with each $0 \leq f_{i} \leq 1$ and where the sum runs from $i=1$ to $i=\mathrm{N}$.

The total NCV from the $N$ projects is then just the sum of all so that

$\mathrm{NCV}=\Sigma \mathrm{NCV}_{i}$,

with

$\mathrm{NCV}_{i}=B_{i}\left(1+f_{i}\left(G_{i}-C_{i}\right) / B_{i}\right) p_{i}\left(1-f_{i} C_{i} / B_{i}\right)\left(1-p_{i}\right)$.

Now, write (Eq. 18) in the form

$f_{k}=1-f_{i}-\Sigma^{\prime} f_{j}$,

where the prime on the summation means exclude the $i$ th and $k$ th terms. Equation (21) shows that the fractions for different projects are interdependent. To obtain the values of the budget fractions for the $N$ projects that maximize $\mathrm{NCV}$, one now differentiates (Eq. 20) with respect to $f_{i}$ taking care to account for the fact that $f_{k}$ is dependent on $f_{i}$ through (Eq. 21) and then one sets the resulting derivative to zero. After some tedious algebra, one finds that the result is

$$
\begin{aligned}
& \mathrm{NPV}_{k}\left(p_{k}\left(G_{k}-C_{k}\right)\left(1+f_{k}\left(G_{k}-C_{k}\right) / B_{k}\right)^{-1}\right. \\
& \left.-\left(1-p_{k}\right) C_{k}\left(1-f_{k} C_{k} / B_{k}\right)^{-1}\right) \\
= & \mathrm{NPV}_{i}\left(p_{i}\left(G_{i}-C_{i}\right)\left(1+f_{i}\left(G_{i}-C_{i}\right) / B_{i}\right)^{-1}\right. \\
& \left.-\left(1-p_{i}\right) C_{i}\left(1-f_{i} C_{i} / B_{i}\right)^{-1}\right) .
\end{aligned}
$$

Note that the left side of Eq. (22) has to do only with the $k$ th prospect, while the right hand side has to do only with the $i$ th prospect. But $i$ and $k$ are arbitrary. The only way Eq. (22) can then be satisfied for all values $i=1,2, \ldots . N$ is when each side is constant. Denote this constant by $H$.

Then for all $k$, one has

$$
\begin{gathered}
\operatorname{NPV}_{k}\left(p_{k}\left(G_{k}-C_{k}\right)\left(1+f_{k}\left(G_{k}-C_{k}\right) / B_{k}\right)^{-1}\right. \\
\left.-\left(1-p_{k}\right) C_{k}\left(1-f_{k} C_{k} / B_{k}\right)^{-1}\right)=H
\end{gathered}
$$

As an aside note that if one were to deal with only one prospect, then $H=0$ and so one reverts automatically to the single prospect situation discussed in "Appendix 2". Equation (23) can be written in a simpler form

$$
\begin{aligned}
& C_{k}\left(G_{k}-C_{k}\right)\left(B_{k} / B_{\max , k}-f_{k}\right)\left(1+f_{k}\left(G_{k}-C_{k}\right) / B_{k}\right)^{p_{k^{-1}}} \\
& \quad\left(1-f_{k} C_{k} / B_{k}\right)^{-p_{k}}=H B_{k^{2}}
\end{aligned}
$$

with $B_{\max , k}=C_{k}\left(G_{k}-C_{k}\right) /\left(p_{k} G_{k}-C_{k}\right)$.

Two mathematical problems arise in attempting to solve Eq. (25). First, one must have determined a value for $\mathrm{H}$ and, second, the highly nonlinear nature of Eq. (25) for $f_{k}$ would seem to preclude an analytic solution, then recourse to numerical methods would seem to be the most appropriate path to follow. In this regard, there exists a numerical procedure available with Excel Solver@ that permits one to set ranges for any and all parameters involved and then the program finds those values that maximize NPV for the all the projects taken together.

Inspection of Eq. (25) indicates that there appears to be a solution with $B_{k} / B_{\max , k}=f_{k}$, but remembering that $\Sigma f_{i}=1$ one then has to have two factors in place for such a solution:

$f_{k}=B_{k} \mathrm{EV}_{k} /\left(C_{k}\left(G_{k}-C_{k}\right)\right) / \Sigma\left(B_{i} \mathrm{EV}_{i} /\left(C_{i}\left(G_{i}-C_{i}\right)\right)\right)$,

with $B_{i}<C_{i}$ for all $N$ projects. At the optimum working interest for each project, one has $B_{i}=C_{i}$ so that the maximum investment is then

$B=\Sigma C_{i}$.

The corresponding fractions are then obtained from Eq. (25) as

$f_{k}=\mathrm{EV}_{k} /\left(G_{k}-C_{k}\right) / \Sigma\left(\mathrm{EV}_{i} /\left(G_{i}-C_{i}\right)\right)$.

\section{Appendix 4}

A risk method when $\mathrm{EV}<0$.

Start with the OWI from the Kelly risking method as

$\mathrm{OWI}=\mathrm{EV} /(G-C)$.

Because one knows that there is a standard deviation, $\sigma$, around the mean $\mathrm{EV}$ given through 
$\sigma= \pm G(p(1-p))^{1 / 2}$,

it follows that OWI will also be uncertain. The linearity of OWI in EV then means that OWI has a standard deviation around the mean given through

$\Sigma= \pm(p(1-p))^{1 / 2} /(1-C / G)$,

so that one can write the range of OWI in the form

Range $=\mathrm{EV} /(G-C) \pm(p(1-p))^{1 / 2} /(1-C / G)$.

When $\mathrm{EV}<0$, then one can still have a positive value for OWI (at one standard deviation) as long as

$G(p(1-p))^{1 / 2} /(C-p G)>1$,

although one should place less and less confidence in such hydrocarbon exploration projects as the left hand side of inequality (D5) approaches unity from above. Projects with the left hand side of inequality (D5) $<1$ should be avoided at the one standard deviation level. This sort of estimation allows negative $\mathrm{EV}$ values to still be considered as valuable projects provided the $\mathrm{EV}$ is not so negative that inequality (D5) is not satisfied.

\section{References}

\section{List A. References directly quoted in text}

Bernoulli D (1954) [1738] Exposition of a new theory on the measurement of risk. Econometrica 22(1):22-36. (JSTOR 1909829)

Cozzolino JM (1977) A simplified utility framework for the analysis of financial risk. In: Econ. Eval. Sympos. Soc. Pet. Eng., Dallas, TX., SPE No. 6, 359

Kelly JL (1956) A new interpretation of information rate. Bell Syst Tech J 35(4):917-926. https://doi.org/10.1002/j.1538-7305.1956. tb03809.x

Lerche I, MacKay JA (1999) Economic risk in hydrocarbon exploration. Academic Press, Waltham

Lerche I, MacKay J (2003) Optimum working interest in hydrocarbon exploration projects: the high gain situation revisited for cost, wealth and fear factors. Energy Explor Exploit 21:381-390

MacKay JA (2015) Kelly versus OWI. In: Gulf coast association of geological societies proceedings

Mian MA (2002) Project Economics and decision analysis: probabilistic models, probabilistic methods. Pennwell Corp., Tulsa

\section{List B. Background references for the fields of gambling and stock market investment}

Adler T, Kritzman M (2006) Mean-variance versus Full-Scale Optimization: in and out of sample. J Asset Manag 7:302-311
Bekaert G, Erb C, Harvey C, Viskanta T, 1998, Distributional characteristics of emerging market returns and asset allocation, J Portfolio Manag 5:102-116

Breiman L (1961) Optimal gambling systems for favorable games. In: Fourth berkeley symposium on probability and statistics 1:65-78

De Brouwer P, Van den Spiegel F (2001) The fqallacy of large numbers revisited: the construction of a utility function that leads to the acceptance of two games, while one is rejected. J Asset Manag $1: 257-266$

Elton E, Gruber M (1974) On the maximization of the geometric mean with lognormal return distribution. Manag Sci 21:483-488

Estrada J (2006) Downside risk in practice. J Appl Corp Financ $18: 117-125$

Estrada J, 2008, Mean-semi-variance optimization: a heuristic approach, J Appl Financ 6:57-72

Estrada J, 2010, Geometric mean maximization: an overlooked portfolio approach? http://www.iese.edu/professors/faculty-directory/ javier-estrada

Fama E, McBeth J (1974) Long-term growth in a short-term market. J Financ 29:857-885

Grauer R (1981) A comparison of growth optimal and mean variance investment policies. J Financ Quant Anal 16:1-21

Hakansson N (1971a) Capital growth and the mean-variance approach to portfolio selection. J Financ Quant Anal 6:517-557

Hakansson N (1971b) Multi-period mean-variance analysis: toward a general theory of portfolio choice. J Financ 26:857-884

Hung J (2010) Betting with the Kelly criterion http://www.math.washi ngton.edu/ morrow/336_10/papers/jane.pdf

Merton R, Samuelson P (1974) Fallacy of the log-normal approximation to optimal portfolio decision-making over many periods. $\mathrm{J}$ Financ Econ 1:67-94

Ophir T (1978) The geometric mean principle revisited. J Bank Financ 2:103-107 (see also the same author in Journal of Banking and Finance 3, 301-303)

Poundstone W (2005) Fortune's formula - the untold story of the scientific betting system that beat the casinos and wall street. Hill and Wang, New York

Rotando L, Thorp EO (1992) The kelly criterion and the stock market. Am Math Month 99:922-931

Thorp EO (1969) Optimal gambling systems for favorable games. Rev L'Inst Int Stat 37:273-293

Thorp EO (1997) The kelly criterion in blackjack, sports betting, and the stock market, paper presented at: In: The 10th international conference on gambling and risk taking

Young W, Trent R (1969) Geometric mean approximations of individual security and portfolio performance. J Financ Quant Anal 4:179-199

Publisher's Note Springer Nature remains neutral with regard to jurisdictional claims in published maps and institutional affiliations. 